\title{
The association between migraine and hospital readmission due to pain after surgery: A hospital registry study
}

2019, Vol. 39(2) 286-295

(C) International Headache Society 2018

Article reuse guidelines:

sagepub.com/journals-permissions DOI: I0.II77/03331024|8786457 journals.sagepub.com/home/cep

(S)SAGE

\author{
Katharina Platzbecker ${ }^{1,2, *}$, Megan Behua Zhang ${ }^{1, *}$, \\ Tobias Kurth ${ }^{3}$, Maira Isabella Rudolph', Katharina Eikermann- \\ Haerter $^{4}$, Rami Burstein ${ }^{2}$, Matthias Eikermann ${ }^{2,5}$ and \\ Timothy Houle'
}

\begin{abstract}
Background: Migraine has been identified as a risk factor of 30-day hospital readmission after surgery. We aimed to further characterize this association examining pain as a potentially migraine-associated, preventable reason for readmission.

Hypothesis: Compared to patients with no migraine, surgical patients with migraine are at increased risk of 30-day hospital readmission with an admitting diagnosis specifying pain.

Methods: This hospital registry study examined I50,7 I0 patients aged 18 years and above, who underwent surgery with general anesthesia and mechanical ventilation between 2007 and 2015 at a tertiary care center and two affiliated community hospitals in Massachusetts, USA.

Results: Migraine was associated with an increased risk of 30-day pain-related readmission after surgery (adjusted odds ratio 1.42 [95\% confidence interval 1.15-1.75]). The association was stronger for migraine with aura (compared to migraine without aura: Adjusted odds ratio 1.69 [95\% confidence interval 1.06-2.70]; compared to no migraine: Adjusted odds ratio 2.20 [95\% confidence interval 1.44-3.37]). The predicted adjusted risk of pain-related 30-day readmissions was 9.1 [95\% confidence interval $5.3-13.0]$ in 1000 surgical patients with migraine with aura and 5.4 [95\% confidence interval 4.2-6.6] in 1000 patients with migraine without aura, compared to 4.2 [95\% confidence interval 3.8-4.5] in 1000 patients with no migraine. Furthermore, migraine was associated with an increased risk of postsurgical 30-day readmission due to a priori defined migraine-related pain (headache or abdominal pain) (adjusted odds ratio I.55 [95\% confidence interval I.20-2.00]).

Conclusion: Patients with migraine undergoing surgery are at increased risk of 30-day hospital readmission due to pain.
\end{abstract}

\section{Keywords}

Migraine, hospital readmission, pain, perioperative, surgery

Date received: 9 January 20I8; revised: 7 April 20I8; 24 May 20I8; accepted: I June 2018

\section{Introduction}

Unplanned hospital readmissions after surgery have been discussed as a metric for performance in perioperative patient care $(1,2)$. In 2013, the costs of a readmission on average were $\$ 3,000$ higher than those of an index admission in patients covered by private insurance and Medicaid (3). The Centers for Medicare and Medicaid Services took measures aiming to curb readmissions, which include the public reporting of readmission rates and financial sanctions for health care institutions with unexpectedly frequent findings. The identification and reduction of readmissions that can be avoided are thought to lower costs as well as enhance patient safety and quality of care (4).
'Department of Anesthesia, Critical Care, and Pain Medicine, Massachusetts General Hospital and Harvard Medical School, Boston, MA, USA

${ }^{2}$ Department of Anesthesia, Critical Care and Pain Medicine, Beth Israel Deaconess Medical Center and Harvard Medical School, Boston, MA, USA

${ }^{3}$ Institute of Public Health, Charité - Universitätsmedizin Berlin, Berlin, Germany

${ }^{4}$ Department of Radiology, Massachusetts General Hospital and Harvard Medical School, Boston, MA, USA

${ }^{5}$ Klinik für Anästhesiologie und Intensivmedizin, Universitätsklinikum Essen, Essen, Germany

*These authors contributed equally to this work.

\section{Corresponding author:}

Matthias Eikermann, Beth Israel Deaconess Medical Center, One Deaconess Road, Boston, MA 02215, USA.

Email: meikerma@bidmc.harvard.edu 
Migraine, and especially migraine with aura, is a risk factor for hospital readmission within 30 days after discharge from a surgical procedure (5). The greater proportion of readmissions might reflect deficits in the perioperative management of patients with migraine $(1,2)$. In order to further characterize this association, we examined potentially migraine-associated, potentially preventable reasons for readmission, which are pain conditions. Today, migraine is recognized as a complex neurological disorder with pathophysiological mechanisms affecting multiple structures of the nervous system. Patients with migraine have been shown to experience an altered perception and processing of pain (6), and allodynia to be common particularly in patients with chronic migraine and migraine with aura (7). Past studies revealed that migraine may be accompanied with non-cephalic pain conditions (8). An association between migraine and abdominal pain in adults has been examined intensely and could be substantiated (9-11).

We hypothesized that surgical patients with a diagnosis of migraine are at increased risk of readmission within 30 days from discharge due to an admitting diagnosis specifying pain, compared to those with no migraine. Furthermore, we predicted that patients with migraine with aura are at even greater risk $(5,7)$. Secondarily, we hypothesized that the association between migraine and readmission due to pain would be stronger when limiting our outcome to a prioridefined migraine-related pain symptoms (headache and abdominal pain) as previously characterized in literature (9-11).

\section{Methods}

This was a hospital-based registry study using prospectively collected electronic patient data on file at Massachusetts General Hospital, a tertiary care facility in Boston, Massachusetts, and two community hospitals affiliated with Partners Healthcare in Massachusetts, USA (Mass General Waltham, Waltham and Mass General/North Shore Center for Outpatient Care, Danvers). All data were derived from three clinical databases, which include the Research Patient Data Registry, the Anesthesia Information Management System and Enterprise Performance Systems Incorporation. We obtained all billing data from the Research Patient Data Registry, a centralized registry for electronic medical chart information specifically designed for research purposes. From the Anesthesia Information Management System, we received information on intraoperative medication and physiological monitoring. The Enterprise Performance System Incorporation is a financial planning institution, and provided data on admission, discharge and readmission details. All patient data were compiled into a single, de-identified database including 178,718 surgical cases from January 2007 until the end of 2015. The protocol of this study was approved by the Institutional Review Board associated with Partners Healthcare (Partners Human Research Committee; protocol number: 2017P000470) with informed consent waived.

We included all patients who had undergone surgery under general anesthesia and mechanical ventilation with postprocedural extubation within the operating room and survived their index hospital stay. Patients younger than 18 years of age and patients with an American Society of Anesthesiologists (ASA) physical classification status of 6 (brain-dead) (12) were excluded. Our final cohort included only patients who had no missing values for the variables used in our analyses.

\section{Definitions of exposure and outcome}

A diagnosis of migraine was determined using ICD-9/10 diagnostic codes (International Classification of Disease, Ninth/Tenth Revision). As migraine is characterized by recurrent attacks over several years or even a lifetime, (13) we considered patients with a billing diagnosis of migraine at any time within our dataset as exposed. Patients who were not billed for an ICD-9/10 diagnosis of migraine were classified as not exposed.

We defined the outcome as readmission due to pain within 30 days after discharge from surgical procedure to a hospital in the Partners Healthcare network. Therefore, we identified patients who were coded for any ICD-9/10 readmission admitting diagnosis specifying pain. The admitting diagnosis is a single diagnosis defined as the main reason for the hospital consultation and is provided by the admitting physician to describe the patient condition upon first arrival to the hospital. It is assigned before further test or examination results are available and commonly stated as a problem or symptom. The admitting diagnosis is both in terms of time and content clearly distinguishable from principal and secondary diagnoses.

\section{Statistical analyses}

All analyses were conducted with predefined endpoints and methods utilizing the statistics software STATA version 13. Two-tailed $p$-values $(p<0.05)$ were considered statistically significant.

Primary analysis. Our primary model estimated odds ratios (OR) with $95 \%$ confidence intervals (CI) using multivariable logistic regression to test the association between migraine and hospital readmission with an 
admitting pain diagnosis within 30 days after discharge from surgery. Therefore, we included the migraine status as indicator variable differentiating between a diagnosis of migraine versus no diagnosis of migraine. We controlled for potentially confounding variables, which were defined a priori based on literature research, pathophysiological and clinical plausibility. These included demographic and baseline data, information on patient history and comorbidities as well as intraoperative parameters. Covariates for our primary model included sex, age, body mass index, ASA physical status (12), Medicare or Medicaid insurance coverage, history of diabetes, substance abuse, chronic obstructive pulmonary disease, coronary artery disease and stroke, sepsis upon index admission, intensive care unit (ICU) stay during index admission, adverse discharge disposition (discharge to nursing facility, long term care or swing bed (skilled nursing facility bed provided by small hospitals)), prescription for opioids within 30 days after surgery, parameters reflecting surgical complexity such as emergent versus non-emergent surgery, inpatient surgery, duration of surgery, work relative value units (14), intraoperative hypotensive minutes (mean arterial pressure $<55 \mathrm{mmHg}$ ), total intraoperative fluid volume (crystalloids and colloids in ratio 1.5:1) (15) and requirement for transfusion of packed red blood cells, total intraoperative neuromuscular blocking agent effective dose and total intraoperative long-acting morphine equivalent dose (without fentanyl) (16).

Adjusted predicted risks of 30-day readmission after surgery due to pain were calculated for patients with no migraine, all patients with migraine, as well as migraine patients with and without aura, respectively, using an average marginal effects model that was based on the primary model.

Secondary analysis. We defined "migraine-related pain" a priori based on published information as a diagnosis of any headache or abdominal pain (9-11). We then built our primary confounder control model for the outcome migraine-related pain.

Sensitivity analyses. We validated our primary regression model for 30-day readmission after surgery due to pain to evaluate the model fit as well as underlying assumptions. We generated a probability score for 30 -day postsurgical readmission due to pain, independent of our exposure migraine, using the covariates of our primary model. We then performed concordance statistics, more specifically the area under the receiver operating characteristic curve (AUC).

To account for the competing risk of death before readmission, we conducted a time-to-event analysis utilizing the Cox proportional hazards regression. We built a model incorporating the time to readmission within the 30-day period after discharge and controlled for the covariates used in the primary analysis. The satisfaction of the proportional hazards assumption was tested and we found no violation.

Please see the online Supplementary material for further sensitivity analyses, such as multiple imputations and redefinition of the exposure migraine utilizing prescriptions for migraine-specific treatment.

Exploratory analyses. Several exploratory analyses were performed post-hoc to further investigate the implications of our results.

To further evaluate our outcome measure, we subdivided the ICD-9 and -10 readmission admitting pain diagnoses, which were reported within our study cohort, by pain location. A logistic regression adjusted for age and gender was performed to determine the association of migraine with each pain subgroup separately.

We examined whether migraine treatment according to current guidelines had an influence on the association between migraine and readmission due to pain. Migraine abortive treatment was defined as a prescription for triptans or ergotamine at any time prior to surgery (17). Prophylactics were defined as a prescription for substances recommended in the American Academy of Neurology and American Headache Society guidelines with evidence level A and B (betablockers, antidepressants, antiepileptics) (18) or onabotulinumtoxin A (19) at any time prior to surgery. We tested for an effect modification by incorporating the interaction terms migraine*migraine abortive treatment and migraine*migraine prophylactic treatment separately into our primary model.

To gain an impression on how pain is treated within our study cohort, specifically in patients with migraine, we investigated the prevalence of opioid utilization after surgery. We defined opioid treatment as a prescription for opioids at any time within 30 days after surgery.

\section{Results}

\section{Patient characteristics}

A total number of 178,718 surgical patients were reviewed for eligibility. The final study cohort was comprised of 150,710 cases. 15,930 cases were excluded due to missing data for any covariates. In all, 12,048 cases were younger than 18 years of age and 30 cases had an ASA physical status of 6 , and therefore met our a priori defined exclusion criteria (Figure 1). Of the 150,710 surgical cases, 11,768 (7.8\%) had an ICD-9/10 diagnosis of any migraine, of whom $1540(13.1 \%)$ cases had a 


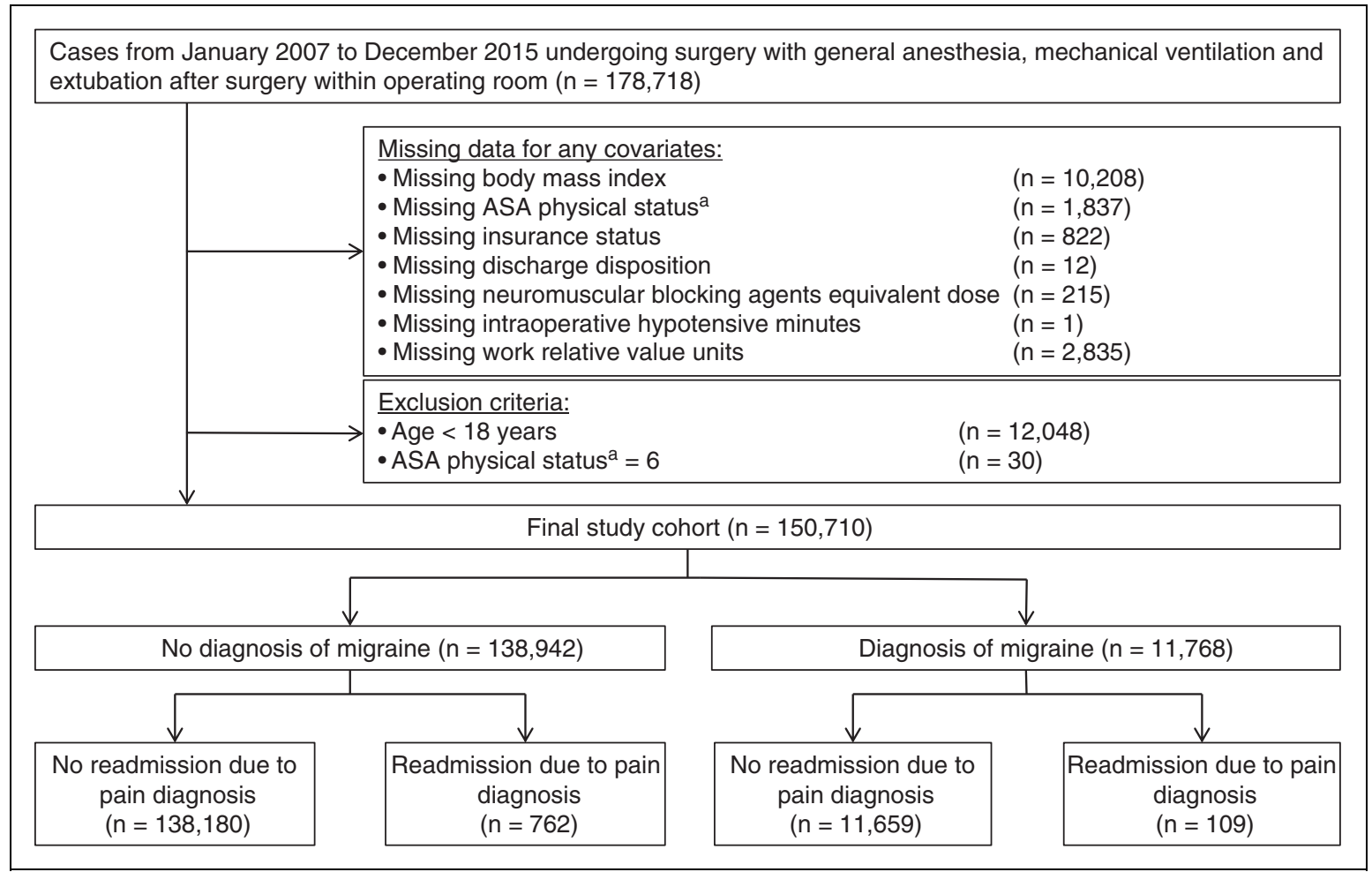

Figure I. Study flow diagram.

${ }^{a}$ American Society of Anesthesiologists physical status classification.

diagnosis of migraine with aura. Overall, patients with migraine were younger and more often female compared to patients without migraine. For the index hospital stay, we observed relatively fewer emergency procedures, ICU stays and adverse discharges among patients with a diagnosis of migraine. Apart from a history of stroke and substance abuse, patients with migraine also showed a lower prevalence of comorbidities (please see Table 1 for further detail).

\section{Primary outcome}

Within our study cohort, $871(0.6 \%)$ patients were readmitted with an admitting diagnosis specifying pain within 30 days after discharge from surgical procedure. Readmitted due to pain were $109(0.9 \%)$ within the group of patients with migraine and $762(0.5 \%)$ within the group of those with no migraine. Multivariable logistic regression revealed a statistically significant association between a diagnosis of migraine and 30-day readmission due to pain after surgery (adjusted OR (aOR) 1.42 [95\% CI 1.15-1.75]), compared to no diagnosis of migraine. There was a numerically stronger association with painrelated readmission for migraine with aura (aOR 2.20 [95\% CI 1.44-3.37]) than for migraine without aura (aOR 1.30 [95\% CI 1.03-1.64]), in comparison to no migraine. The stronger association could be substantiated as being statistically significant when directly comparing migraine with aura to migraine without aura as baseline (aOR 1.69 [95\% CI 1.06-2.70]) (please see Table 2 for primary results). In terms of adjusted predicted risk, overall 5.9 [95\% CI 4.7-7.1] out of 1000 surgical patients with migraine would be readmitted to the hospital due to pain. With an adjusted predicted probability of 9.1 [95\% CI 5.3-13.0] out of 1000, patients with migraine with aura were at higher risk than patients with migraine without aura (5.4 [95\% CI 4.2-6.6] of 1000). Patients with no diagnosis of migraine showed an adjusted predicted risk for 30-day pain-related readmission after surgery of 4.2 [95\% CI 3.8-4.5] out of 1000 .

\section{Secondary outcomes}

In all, $68.8 \%$ of patients with migraine readmitted due to pain after surgery had a migraine-related pain admitting diagnosis as a priori defined (headache or abdominal pain). Migraine was associated with 30-day hospital readmission after surgery due to migrainerelated pain (aOR 1.55 [95\% CI 1.20-2.00]), which represents a numerically stronger association than for our primary outcome.

\section{Sensitivity analyses}

The AUC for the probability of 30-day readmission due to pain after surgery obtained through our 
Table I. Description of study cohort by migraine status and covariates.

\begin{tabular}{|c|c|c|c|c|}
\hline & $\begin{array}{l}\text { Entire cohort } \\
\mathrm{n}=150,710\end{array}$ & $\begin{array}{l}\text { No migraine } \\
\mathrm{n}=138,942\end{array}$ & $\begin{array}{l}\text { Migraine without } \\
\text { aura } \\
n=10,228\end{array}$ & $\begin{array}{l}\text { Migraine with } \\
\text { aura } \\
n=1540\end{array}$ \\
\hline \multicolumn{5}{|l|}{ Demographics and baseline information ${ }^{a}$} \\
\hline Age & $54.44 \pm 16.52$ & $54.94 \pm 16.56$ & $48.86 \pm 14.83$ & $46.35 \pm|4.2|$ \\
\hline Male & $68,702(45.6 \%)$ & $66,425(47.8 \%)$ & 1991 (19.5\%) & $286(18.6 \%)$ \\
\hline Female & $82,008(54.4 \%)$ & 72,517 (52.2\%) & $8237(80.5 \%)$ & 1254 (8I.4\%) \\
\hline Body mass index & $28.45 \pm 6.99$ & $28.4 I \pm 6.91$ & $28.96 \pm 7.90$ & $28.32 \pm 7.43$ \\
\hline $\begin{array}{l}\text { American Society of Anesthesiologists } \\
\text { physical status }\end{array}$ & $2.00(2.00,3.00)$ & $2.00(2.00,3.00)$ & $2.00(2.00,3.00)$ & $2.00(2.00,3.00)$ \\
\hline Medicare or Medicaid insurance coverage & $58,075(38.5 \%)$ & $53,846(38.8 \%)$ & $3674(35.9 \%)$ & $555(36.0 \%)$ \\
\hline Emergent surgery & $6066(4.0 \%)$ & $5653(4.1 \%)$ & $364(3.6 \%)$ & $49(3.2 \%)$ \\
\hline \multicolumn{5}{|l|}{ Characteristics of index admission ${ }^{a}$} \\
\hline Inpatient & $94,58 \mathrm{I}(62.8 \%)$ & $87,373(62.9 \%)$ & $6328(61.9 \%)$ & $880(57.1 \%)$ \\
\hline Intensive care unit stay during index admission & $14,384(9.5 \%)$ & $13,635(9.8 \%)$ & $658(6.4 \%)$ & $91(5.9 \%)$ \\
\hline Sepsis upon index admission & 1897 (I.3\%) & $181 \mid(1.3 \%)$ & $73(0.7 \%)$ & $13(0.8 \%)$ \\
\hline Adverse discharge' & $10,522(7.0 \%)$ & $10,039(7.2 \%)$ & $433(4.2 \%)$ & $50(3.2 \%)$ \\
\hline Hospital length of stay & $5.27 \pm 8.34$ & $5.33 \pm 8.44$ & $4.52 \pm 6.75$ & $4.73 \pm 9.01$ \\
\hline \multicolumn{5}{|l|}{ Patient history ${ }^{\mathrm{a}}$} \\
\hline Diabetes mellitus & $21,494(14.3 \%)$ & $19,982(14.4 \%)$ & $1313(12.8 \%)$ & $199(12.9 \%)$ \\
\hline Hypertension & $64,049(42.5 \%)$ & $59,502(42.8 \%)$ & $3954(38.7 \%)$ & $593(38.5 \%)$ \\
\hline Coronary artery disease & 17,975 (II.9\%) & $16,978(12.2 \%)$ & $864(8.4 \%)$ & $133(8.6 \%)$ \\
\hline Congestive heart failure & 9468 (6.3\%) & $8939(6.4 \%)$ & $454(4.4 \%)$ & $75(4.9 \%)$ \\
\hline Chronic obstructive pulmonary disease & $10,297(6.8 \%)$ & $9611(6.9 \%)$ & $603(5.9 \%)$ & $83(5.4 \%)$ \\
\hline Stroke & $5502(3.7 \%)$ & 4954 (3.6\%) & $452(4.4 \%)$ & $96(6.2 \%)$ \\
\hline Substance abuse & $20,05 \mathrm{I}(13.3 \%)$ & $18,264(13.1 \%)$ & $1550(\mid 5.2 \%)$ & 237 (I5.4\%) \\
\hline \multicolumn{5}{|l|}{ Intra- and perioperative characteristics ${ }^{\mathrm{a}}$} \\
\hline Work RVUs ${ }^{2}$ & $14.45(8.05,21.86)$ & $14.45(8.05,21.87)$ & $14.30(8.89,21.84)$ & $12.13(7.50,18.46)$ \\
\hline Hypotensive minutes & $0.00(0.00,2.00)$ & $0.00(0.00,2.00)$ & $0.00(0.00,2.00)$ & $0.00(0.00,1.00)$ \\
\hline Long-acting opioids $\mathrm{ED}^{3}$ & $3.40(0.00,7.93)$ & $3.40(0.00,7.93)$ & $4.00(0.00,8.50)$ & $3.40(0.00,8.50)$ \\
\hline Intraoperative fluids $(\mathrm{ml})^{4}$ & $\begin{array}{l}\mathrm{I}, 200.00(800.00 \\
2000.00)\end{array}$ & $\begin{array}{l}1200.00(800.00 \\
2000.00)\end{array}$ & $\begin{array}{l}1250.00(800.00 \\
2000.00)\end{array}$ & $\begin{array}{l}\mathrm{I} 100.00(750.00 \\
2000.00)\end{array}$ \\
\hline NDNMBAs ED ${ }^{5}$ & $2.28(0.8 I, 3.6 I)$ & $2.28(0.83,3.62)$ & $2.26(0.70,3.64)$ & $2.17(0.00,3.33)$ \\
\hline Required intraoperative packed red blood cells & $5053(3.4 \%)$ & $4808(3.5 \%)$ & $221(2.2 \%)$ & $24(1.6 \%)$ \\
\hline Opioids prescribed within $30 \mathrm{~d}$ after surgery & $95,346(63.3 \%)$ & $87,74 \mid(63.1 \%)$ & $6639(64.9 \%)$ & $966(62.7 \%)$ \\
\hline
\end{tabular}

${ }^{a}$ Values provided as frequency (prevalence in \%) or mean \pm standard deviation or median (interquartile range [25th to 75 th percentile], values separated by comma).

'Discharge to nursing facility, long term care or swing bed (skilled nursing facility bed provided by small hospitals).

${ }^{2}$ Work relative value units based on surgical CPT (Current Procedural Terminology) codes.

${ }^{3}$ Morphine oral equivalent dose of long-acting opioids (meperidine, morphine, methadone, hydromorphone).

${ }^{4}$ Crystalloids ( $0.9 \%$ normal saline, lactated Ringer's) and colloids (hextend, albumin) in crystalloid-to-colloid ratio 1.5:I.

${ }^{5}$ Multiples of the $95 \%$ effective dose equivalent of intraoperatively administered neuromuscular blocking agents.

covariate model used in the primary analysis measured 0.71. The hazard ratio (HR) resulting from the Cox regression model (HR 1.43 [95\% CI 1.16-1.76]) confirmed the results of the primary analysis.

For the results of further sensitivity analyses please see the Supplementary material.

\section{Exploratory analyses}

After subdividing the outcome by pain location (headache, abdominal pain, postoperative pain, chest pain or painful respiration, pain in limb or joint, backache, neoplasm-related pain, atherosclerosis-related pain, other pain), a significant association with the exposure 
Table 2. Association between migraine and 30 day-readmission due to pain after surgery.

\begin{tabular}{|c|c|c|c|c|c|}
\hline & Prevalence $(\%)^{c}$ & \multirow[b]{2}{*}{$\mathrm{OR}^{\mathrm{a}}(95 \% \mathrm{Cl})$} & \multirow[b]{2}{*}{$p$-value ${ }^{a}$} & \multicolumn{2}{|l|}{ Adjusted $^{b}$} \\
\hline & $\begin{array}{l}30 \text { day-readmission after } \\
\text { surgery due to pain }\end{array}$ & & & $\mathrm{OR}^{\mathrm{a}}(95 \% \mathrm{Cl})$ & $p$-value \\
\hline Any migraine & $0.93 \%$ & $1.70(1.39-2.07)$ & $<0.001$ & $1.42(1.15-1.75)$ & 0.001 \\
\hline Migraine without aura & $0.84 \%$ & $1.54(1.23-1.92)$ & $<0.001$ & $1.30(1.03-1.64)$ & 0.026 \\
\hline Migraine with aura & $1.49 \%$ & $2.75(1.81-4.18)$ & $<0.001$ & $2.20(1.44-3.37)$ & $<0.001$ \\
\hline No migraine & $0.55 \%$ & 1.00 & & 1.00 & \\
\hline
\end{tabular}

${ }^{a}$ Results of logistic regression ("pain readmission" vs. "no pain readmission", reference: "No pain readmission") for each migraine group compared to "no migraine".

besults of multivariable logistic regression adjusted for a priori defined covariates (as shown in Table I).

"Within "migraine status" group.

migraine could be found only for headache (aOR 2.73 [95\% CI 1.62-4.60]) and abdominal pain (aOR 1.56 [95\% CI 1.17-2.08]) (Table 3).

In all, $2714(23.1 \%)$ patients with migraine had received a prescription for triptans or ergotamine any time prior to surgery. Such a migraine abortive treatment numerically modified the risk of 30-day readmission due to pain after surgery among patients with migraine, but the effect was not statistically significant (interaction term: aOR $0.48 \quad\left[\begin{array}{llll}9 \% & \text { CI } & 0.20-1.13\end{array}\right]$, $p=0.091)$.

A total of $5689(48.3 \%)$ cases among the group of patients with migraine had a prescription for migraine prophylactic medication. Prophylactic treatment did not show a significant effect on the association between migraine and 30-day postsurgical readmission due to pain $(p$ for effect modification $=0.45$ )

A total of $10,230(86.9 \%)$ of the patients with migraine had received an opioid prescription at any time prior to surgery. The screening for opioid prescriptions within 30 days after surgery revealed that 7605 $(64.6 \%)$ of the patients with migraine were exposed to such medication.

\section{Discussion}

We observed a significant association between migraine and 30-day readmission after surgery with an admitting diagnosis specifying pain. Although patients with migraine tended to be younger and showed fewer indicators for a severe course of the index hospital stay, they were at increased risk to be readmitted due to pain within 30 days after discharge from surgery (aOR 1.42 [95\% CI 1.15-1.75]). Patients with migraine with aura were at significantly higher risk compared to patients with migraine without aura (aOR $1.69[95 \%$ CI 1.06-2.70]). Furthermore, patients with migraine were at significantly higher risk of postsurgical 30-day readmission due to migraine-related pain (headache or abdominal pain) (9-11) (aOR $1.55 \quad[95 \%$ CI 1.20-2.00]). A potential mitigating effect of migraine abortive treatment on the observed association, suggested by our results, is to be further investigated.

Patients with migraine are at increased risk of 30-day hospital readmission after surgery (5). Our study shows that postoperative pain conditions are contributing to migraine-associated 30-day hospital readmissions after surgery. Of the readmitted patients with a diagnosis of migraine, $10.8 \%$ had a pain-related admitting diagnosis, and we speculate that some of these pain-related postsurgical readmissions may be preventable.

The locations of pain symptoms observed at readmission within 30 days after surgery are presented in Table 3. Among the 109 readmitted subjects with migraine and with a pain diagnosis, $81 \%$ suffered from pain located in the surgical field. An increased vulnerability of patients with migraine to experiencing chronic pain in the vicinity of the surgical field has been reported after thoracic, abdominal, urological, gynecological and orthopedic surgery, including amputations (20). Pain pathways may be altered in migraine patients, resulting in a phenotype that is hyperexcitable, hypersensitive, and hyperresponsive due to an impairment of pain modulation (6).

In our study, we also observed an association between migraine and readmission of migraine-related pain (headache or abdominal pain) that cannot directly be explained by the surgical trauma. Migraine was individually associated with postsurgical 30-day readmission for both of these types of pain. It is well known that patients with migraine are vulnerable in the perioperative phase with the headache occurring within hours and up to five days after surgery (21-23). Our data confirm these findings and add the information that both headache and abdominal pain after surgery are a mechanism of readmission to the hospital.

Abdominal pain is associated with migraine (9-11). In our study, many patients were included after 
Table 3. Frequencies and prevalence of readmission pain diagnoses within study cohort. ICD codes as found within study cohort.

\begin{tabular}{|c|c|c|c|}
\hline Readmission pain diagnoses & $\mathrm{n}$ & Prevalence & ICD code \\
\hline Total & 871 & $100.00 \%$ & \\
\hline Abdominal pain & 434 & $49.83 \%$ & \\
\hline Unspecified site & 264 & & 789.00, RI0.9 \\
\hline Other specified site, multiple sites & 93 & & 789.09 \\
\hline Right upper quadrant & 29 & & 789.0I, RI0.1I \\
\hline Right lower quadrant & 15 & & 789.03, RI0.3I \\
\hline Epigastric & 15 & & 789.06 \\
\hline Left lower quadrant & 10 & & 789.04 \\
\hline Generalized & 3 & & 789.07 \\
\hline Flatulence, eructation and gas pain & 2 & & 787.3 \\
\hline Anal or rectal pain & 2 & & 569.42 \\
\hline Lower abdominal pain, unspecified & I & & RI0.30 \\
\hline Chest pain or painful respiration & 114 & $13.09 \%$ & \\
\hline Unspecified & 94 & & 786.50, R07.9 \\
\hline Other chest pain & 16 & & 786.59 \\
\hline Painful respiration, chest pain on breathing & 4 & & 786.52, R07.I \\
\hline Headache & 93 & $10.68 \%$ & \\
\hline Headache & 90 & & 784.0, R5I \\
\hline Other forms of migraine, w/o mention of intractable migraine & I & & 346.8 \\
\hline Migraine, unspecified w/o mention of intractable migraine & I & & 346.9 \\
\hline Hemicrania continua & I & & 339.41 \\
\hline Pain in limb or joint & 79 & $9.07 \%$ & \\
\hline Pain in limb & 42 & & 729.5 \\
\hline Pain in joint involving lower leg & 14 & & $719.46, M 79.669$ \\
\hline Pain in joint involving pelvis & 12 & & 719.45 \\
\hline Pain in joint involving shoulder & 2 & & 719.41 \\
\hline Pain in left hip & 2 & & $M 25.552$ \\
\hline Pain in joint involving ankles & I & & 719.47 \\
\hline Pain in right elbow & I & & $M 25.521$ \\
\hline Pain in right knee & I & & $M 25.56 \mathrm{I}$ \\
\hline Pain in unspecified knee & I & & $M 25.569$ \\
\hline Pain in right leg & I & & M79.604 \\
\hline Pain in right thigh & I & & M79.65I \\
\hline Arthralgia of the temporomandibular joint & I & & 524.62 \\
\hline Backache & 51 & $5.86 \%$ & \\
\hline Lumbago & 19 & & 724.2 \\
\hline Unspecified & 19 & & 724.5 \\
\hline Cervicalgia & 9 & & 723.I, M54.2 \\
\hline Low back pain & 3 & & M54.5 \\
\hline Sciatica & I & & 724.3 \\
\hline Postoperative pain & 42 & $4.82 \%$ & \\
\hline Other acute postoperative/postprocedural pain & 37 & & $338.18, \mathrm{G} 89.18$ \\
\hline Other chronic postoperative pain & 4 & & 338.28 \\
\hline Acute post-thoracotomy pain & I & & \\
\hline
\end{tabular}


Table 3. Continued.

\begin{tabular}{lccc}
\hline Readmission pain diagnoses & $\mathrm{n}$ & Prevalence & ICD code \\
\hline Neoplasm related pain & 28 & $3.21 \%$ & \\
\hline Acute and chronic & 28 & $\mathrm{G} 89.3,338.3$ \\
Atherosclerosis related pain & 14 & $1.61 \%$ & \\
\hline Atherosclerosis of & 14 & 440.22 \\
$\quad$ extremities with rest pain & & & \\
Other pain & 16 & $1.84 \%$ & \\
\hline Other acute pain & 3 & & 440.22 \\
Other chronic pain & 8 & 338.29 \\
Chronic pain syndrome & $\mathrm{I}$ & 338.4 \\
Trigeminal neuralgia & $\mathrm{I}$ & 350.1 \\
Mastodynia & $\mathrm{I}$ & 611.71 \\
Polymyalgia rheumatica & $\mathrm{I}$ & 725 \\
Generalized pain & $\mathrm{I}$ & 780.96 \\
\hline
\end{tabular}

abdominal surgery such as general surgical (16.4\%), gynecological $(7.6 \%)$ or urological $(9.0 \%)$ procedures but the association between migraine and readmission for abdominal pain was not modified by abdominal surgery (Supplementary material). Even when excluding patients with abdominal surgery, the association of migraine and abdominal pain remained robust.

There are migraine comorbidities that are themselves associated with unique pain symptoms such as fibromyalgia and anxiety diseases, which may also affect healthcare utilization (24-26). In our study, the observed association between migraine and painrelated readmission remained stable and statistically significant when testing for an effect modification by psychiatric and chronic pain comorbidities. These results substantiate migraine to be the driver for the observed association and a potential target point for measures to prevent readmissions due to pain.

Sufficient pain treatment may be an obvious strategy to prevent pain-related hospital consultations. When compared within our group of patients with migraine, prescriptions for migraine abortives showed the lowest $(23.1 \%)$ and those for opioids the highest prescription rate $(86.9 \%)$. We evaluated if migraine-specific abortive medications (triptans and ergotamine) mitigated the migraine-associated vulnerability for 30-day readmission due to pain after surgery and found a nonsignificant trend $(p<0.1)$ towards effect modification. Based on this observation and the fraction of patients who received a migraine abortive treatment, we estimated that in order to conduct an adequately powered study (1-beta $>0.8$, alpha-error $=0.05$ ), about 43,000 surgical patients with migraine would need to be enrolled. There are data from the emergency medicine literature that encourage us to consider such a study with the endpoint 30-day readmission. Deficient Emergency Department (ED) discharge management with lacking migraine-specific prescriptions as well as missing guidance and schedules for follow-up consultations have been described as a reason for ED revisit $(27,28)$. Data on hospital readmission prevention show that the arrangement of a community-based primary care liaison, patient education and medication reconciliation are important strategies (29). Future studies may focus on the creation of an optimal pathway that helps prevent hospital readmissions for pain in patients with migraine.

A total of $64.6 \%$ of patients with migraine received an opioid prescription within 30 days after surgery. In migraine patients, increasing use of opioids (ascending order: Never use, previous use, current use, current use with probable dependence) is associated with an increase in migraine attack frequency and headache-related disability (30). Intraoperative opioid dose is a risk factor for 30-day hospital readmission (16). Furthermore, opioid use leads to an increase in revisits when applied in the ED as abortive migraine treatment (31). In our study, $71.0 \%$ of all patients readmitted with a headache diagnosis had been exposed to opioids within 30 days after surgery, including $68.4 \%$ of patients with migraine readmitted due to headache. In accordance with this observation, opioid utilization is associated with an increased risk for medication-overuse headache, to which migraine patients are specifically vulnerable (32).

The strengths of this study were particularly attributable to the large number of surgical cases analyzed, including both inpatient and outpatient. No specific surgical disciplines or procedures were excluded. The wide variety of surgeries and settings, which included a large, urban university hospital as well as community in- and 
outpatient facilities, render our results generalizable to further patient populations. Finally, the extensive clinical database enabled us to adjust for a large number of potentially confounding variables.

There are limitations to be considered when evaluating our findings. As this was a project on administrative data, the classification of our exposure and outcome variables was based on billing information. ICD codes are susceptible to misclassification. However, we made efforts to analyze the consequences of headache misdiagnoses and failed to identify evidence of a differential bias by headache misclassification. Our data suggest that the association of any headache including migraine with pain-related readmission is most likely driven by migraine (Supplementary material). However, it is not possible to rule out an effect of diagnosis inaccuracy on the observed association between migraine and readmission due to pain. Treatment information was obtained through prescription data. There were no details on the indication and actual individual intake available. A wide variety of possible indications and a mismatch between prescriptions and utilization have to be taken into account, especially regarding prophylactic treatment (33). Our study did not capture those patients readmitted to a hospital outside the wide range of Partners Healthcare acute care facilities (Supplementary material). Available data on trauma patients, however, suggest that the great majority, more specifically three quarters, of patients or primary care providers choose the same hospital when consulting an ED after discharge (34). Furthermore, our readmission data is comparable to the range reported in national data (35). In addition, a sensitivity analysis conducted only in patients with documented follow-up visits at Partners Healthcare during the 30-day period showed reassuring results. Therefore, we reasonably consider the number of readmissions to hospitals outside the Partners Healthcare network to be low.

In summary, our data demonstrate a significant association between migraine and 30-day readmission after surgery for pain that included migraine-associated pain such as headache and abdominal pain.

\section{Clinical implications}

- Patients with a diagnosis of migraine are at increased risk of 30-day hospital readmission after surgery due to suffering from pain.

- A potential mitigating effect by migraine abortive treatment on the association between migraine and postsurgical readmission due to pain is to be further investigated in future projects.

\section{Declaration of conflicting interests}

The authors declared the following potential conflicts of interest with respect to the research, authorship, and/or publication of this article: MERCK: Matthias Eikermann has received investigator-initiated study support and is serving on the advisory board for sugammadex. Tobias Kurth has received honoraria from Lilly for providing methodological advice, from Novartis for a lecture on neuroepidemiology, and from the BMJ for editorial services. He has provided methodological consultancy to CoLucid for which the Charité has received unrestricted funds.

\section{Funding}

The authors disclosed receipt of the following financial support for the research, authorship, and/or publication of this article: This research received no specific grant from any funding agency in the public, commercial or not-for-profit sectors. Matthias Eikermann receives funding from philanthropic donor Dr. Jeffrey Buzen.

\section{References}

1. Krumholz HM, Wang K, Lin Z, et al. Hospital-readmission risk - isolating hospital effects from patient effects. $N$ Engl J Med 2017; 377: 1055-1064.
2. Tsai TC, Joynt KE, Orav EJ, et al. Variation in surgicalreadmission rates and quality of hospital care. $N$ Engl $J$ Med 2013; 369: 1134-1142.

3. Barrett ML, Wier LM, Jiang HJ, et al. All-cause readmissions by payer and age, 2009-2013: HCUP Statistical Brief \#199. Agency for Healthcare Research and Quality, http:// www.hcup-us.ahrq.gov/reports/statbriefs/sb199-

Readmissions-Payer-Age.pdf (2015, accessed 10 December 2017).

4. AHA (American Hospital Association). TrendWatch. Examining the drivers of readmissions and reducing unnecessary readmissions for better patient care, http:// www.aha.org/research/reports/tw/11sep-tw-readmissions. pdf (2011, accessed 10 October 2017).

5. Timm FP, Houle TT, Grabitz SD, et al. Migraine and risk of perioperative ischemic stroke and hospital readmission: Hospital based registry study. BMJ 2017; 356: 16635.

6. Burstein R, Noseda R and Borsook D. Migraine: Multiple processes, complex pathophysiology. J Neurosci 2015; 35: 6619-6629.

7. Aguggia M. Allodynia and migraine. Neurol Sci 2012; 33: 9.

8. Scher AI, Buse DC, Fanning KM, et al. Comorbid pain and migraine chronicity: The Chronic Migraine Epidemiology and Outcomes Study. Neurology 2017; 89: 461-468. 
9. Kurth T, Holtmann G, Neufang-Huber J, et al. Prevalence of unexplained upper abdominal symptoms in patients with migraine. Cephalalgia 2006; 26: 506-510.

10. Roberts JE and deShazo RD. Abdominal migraine, another cause of abdominal pain in adults. Am J Med 2012; 125: 1135-1139.

11. Evans RW and Whyte C. Cyclic vomiting syndrome and abdominal migraine in adults and children. Headache 2013; 53: 984-993.

12. ASA (American Society of Anesthesiologists). ASA Physical Status Classification System, https://www.asahq. org/resources/clinical-information/asa-physical-status-classification-system (2014, accessed 4 January 2018).

13. Lipton RB and Silberstein SD. Episodic and chronic migraine headache: Breaking down barriers to optimal treatment and prevention. Headache 2015; 2: 103-122.

14. Davenport DL, Henderson WG, Khuri SF, et al. Preoperative risk factors and surgical complexity are more predictive of costs than postoperative complications: A case study using the National Surgical Quality Improvement Program (NSQIP) database. Ann Surg 2005; 242: 463-468.

15. Shin $\mathrm{CH}$, Long DR, McLean D, et al. Effects of intraoperative fluid management on postoperative outcomes: A hospital registry study. Ann Surg 2017; 267: 1084-1092.

16. Long D, Friedrich S and Scheffenbichler F. Association between intraoperative opioid administration and 30-day readmission: A prespecified analysis of registry data from a healthcare network in New England. Brit J Anesthesia 2018; 120: 1090-1102.

17. Silberstein SD. Practice parameter: Evidence-based guidelines for migraine headache (an evidence-based review): Report of the Quality Standards Subcommittee of the American Academy of Neurology. Neurology 2000; 55: 754-762.

18. AAN (American Academy of Neurology). Update: Pharmacologic treatment for episodic migraine prevention in adults, https://www.aan.com/Guidelines/Home/GetGuide lineContent/545 (2012, accessed 22 September 2017).

19. AAN (American Academy of Neurology). Practice guideline update summary: Botulinum neurotoxin for the treatment of blepharospasm, cervical dystonia, adult spasticity, and headache, https://www.aan.com/Guide lines/Home/GetGuidelineContent/737 (2016, accessed 22 September 2017).

20. Macrae WA. Chronic pain after surgery. $\mathrm{Br} J$ Anaesth 2001; 87: 88-98.

21. Hannington-Kiff JG. Headache before and after operation in gynaecological patients. Br J Anaesth 1969; 41: 401-406.
22. McDowell SA, Dundee JW and Pandit SK. Para-anaesthetic headache in female patients. Anaesthesia 1970; 25: 334-340.

23. Matsota PK, Christodoulopoulou TC, Batistaki CZ, et al. Factors associated with the presence of postoperative headache in elective surgery patients: A prospective single center cohort study. J Anesth 2017; 31: 225-236.

24. Antonaci F, Nappi G, Galli F, et al. Migraine and psychiatric comorbidity: A review of clinical findings. $J$ Headache Pain 2011; 12: 115-125.

25. Marcus DA, Bernstein C and Rudy TE. Fibromyalgia and headache: An epidemiological study supporting migraine as part of the fibromyalgia syndrome. Clin Rheumatol 2005; 24: 595-601.

26. Boscardin CK, Gonzales R, Bradley KL, et al. Predicting cost of care using self-reported health status data. $B M C$ Health Serv Res 2015; 15: 406.

27. Gupta MX, Silberstein SD, Young WB, et al. Less is not more: Underutilization of headache medications in a university hospital emergency department. Headache 2007; 47: 1125-1133.

28. Nijjar SS, Pink L and Gordon AS. Examination of migraine management in emergency departments. Pain Res Manag 2011; 16: 183-186.

29. Jack BW, Chetty VK, Anthony D, et al. A reengineered hospital discharge program to decrease rehospitalization: a randomized trial. Ann Intern Med 2009; 150: 178-187.

30. Buse DC, Pearlman SH, Reed ML, et al. Opioid use and dependence among persons with migraine: Results of the AMPP study. Headache 2012; 52: 18-36.

31. Young N, Silverman D, Bradford H, et al. Multicenter prevalence of opioid medication use as abortive therapy in the emergency department treatment of migraine headaches. Am J Emerg Med 2017; 16: 30454-30450.

32. Lipton RB and Bigal ME. Opioid therapy and headache: A cause and a cure.

33. Hepp Z, Dodick DW, Varon SF, et al. Persistence and switching patterns of oral migraine prophylactic medications among patients with chronic migraine: A retrospective claims analysis. Cephalalgia 2017; 37: 470-485.

34. Malhotra AK, Martin N, Jacoby M, et al. What are we missing: Results of a 13-month active follow-up program at a level I trauma center. J Trauma 2009; 66: 1696-1702.

35. Gupta A, Nizamuddin J, Elmofty D, et al. Opioid abuse or dependence increases 30-day readmission rates after major operating room procedures: A national readmissions database study. Anesthesiology 2018; 128: 880-890. 


\section{DuEPublico}

Duisburg-Essen Publications online

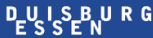

offen im Denken

This text is made available via DuEPublico, the institutional repository of the University of Duisburg-Essen. This version may eventually differ from another version distributed by a commercial publisher.

DOI: $\quad 10.1177 / 0333102418786457$

URN： urn:nbn:de:hbz:464-20210510-162339-3

This publication is with permission of the rights owner freely accessible due to an Alliance licence and a national licence (funded by the DFG, German Research Foundation) respectively.

(C) International Headache Society 2018. All rights reserved. 